\title{
Effect of probiotic and vitamin D supplementation on markers of vitamin D status and bone turnover in healthy adults
}

\author{
T. R. Hill ${ }^{1}$, L. Brennan ${ }^{4}$, A. O’Connor ${ }^{4}$, L. Scully ${ }^{1}$, S. Healy ${ }^{1}$, A. O’Sullivan ${ }^{4}$, B. Mion ${ }^{4}$, G. Dawson ${ }^{1}$, \\ S. Kaluskar ${ }^{4}$, M. J. Gibney ${ }^{4}$, F. Shanahan ${ }^{2,3}$ and K. D. Cashman ${ }^{1,2}$ \\ ${ }^{1}$ Department of Food and Nutritional Sciences, ${ }^{2}$ Department of Medicine and ${ }^{3}$ Alimentary Pharmabiotic Centre, \\ University College, Cork and ${ }^{4}$ Institute of Food and Health, University College Dublin, Dublin 4
}

There is some in vitro evidence that probiotic bacteria may influence Ca uptake in the intestine ${ }^{(1)}$, possibly through an interaction with vitamin D endocrine system ${ }^{(2)}$, with potential benefit for bone turnover. However, the effect of probiotic bacteria on bone turnover has not been investigated in human subjects. The objective of the present study was to examine the effect of 4-week supplementation with probiotics and vitamin D on serum 25-hydroxyvitamin D $(\mathrm{S}-25(\mathrm{OH}) \mathrm{D})$, parathyroid hormone $(\mathrm{PTH})$ and biochemical markers of bone turnover in healthy adults.

In a randomised double-blind placebo-controlled trial conducted in Dublin and Cork, 147 subjects aged between 18-63 years (seventy men and seventy-seven women) were randomised to receive daily for 4 weeks at two different intervention periods (November 2007 and April 2008): $15 \mu \mathrm{g}$ cholecalciferol and $1 \times 10^{9}$ Lactobacillus Salivarius, UCC 118 (probiotic); $15 \mu \mathrm{g}$ cholecalciferol and probiotic placebo; cholecalciferol placebo and probiotic; cholecalciferol placebo and probiotic placebo. S-25(OH)D concentrations and serum concentrations of PTH, osteocalcin (OC), bone-specific alkaline phosphatase (BAP) as well as urinary concentrations of N-telopeptides of type I collagen (NTx) were assessed at baseline and post intervention using commercially-available ELISA kits. One-way between-groups analysis of covariance (ANCOVA) was used to examine the effect of treatment group on post-intervention biochemical variables while including age, gender, season of blood draw and baseline biochemical concentrations as covariates.

\begin{tabular}{|c|c|c|c|c|c|c|c|c|c|}
\hline & \multicolumn{8}{|c|}{ Treatment group } & \multirow[b]{3}{*}{$P^{*}$} \\
\hline & \multicolumn{2}{|c|}{$\begin{array}{l}\text { Vitamin } \mathrm{D} \text { and } \\
\text { probiotic ( } \begin{array}{l}n \\
39)\end{array}\end{array}$} & \multicolumn{2}{|c|}{$\begin{array}{c}\text { Vitamin } \mathrm{D} \text { and probiotic } \\
\text { placebo }(n 36)\end{array}$} & \multicolumn{2}{|c|}{$\begin{array}{c}\text { Vitamin D placebo } \\
\text { and probiotic }\left(\begin{array}{l}n \\
\text { 36) }\end{array}\right.\end{array}$} & \multicolumn{2}{|c|}{ Placebo (n 36) } & \\
\hline & Mean & Range & Mean & Range & Mean & Range & Mean & Range & \\
\hline \multicolumn{10}{|c|}{ S-25(OH)D (nmol/l) } \\
\hline Pre & 58.4 & $24.5-132$ & 61.1 & $22.3-104$ & 55.3 & $22.1-201$ & 45.0 & $27.2-111$ & \\
\hline Post & $75.1^{\mathrm{a}}$ & $39.3-143$ & $76.3^{\mathrm{a}}$ & $40.9-107$ & $51.8^{\mathrm{b}}$ & $25.6-264$ & $46.2^{\mathrm{b}}$ & $27.1-91.1$ & $<0.001$ \\
\hline \multicolumn{10}{|c|}{ S-PTH (pg/ml) } \\
\hline Pre & 75.4 & $40.9-150$ & 82.4 & $34.0-171$ & 83.3 & $43.1-172$ & 82.7 & $40.7-154$ & \\
\hline Post & 66.6 & $36.6-130$ & 85.7 & $44.0-167$ & 83.1 & $34.3-190$ & 74.5 & $34.5-148$ & 0.160 \\
\hline \multicolumn{10}{|c|}{ Urinary NTx (nm BCE) } \\
\hline Pre & 416 & $143-1971$ & 479 & $67-2070$ & 404 & $163-5095$ & 460 & $152-2120$ & \\
\hline Post & 509 & $170-2327$ & 494 & $82-2979$ & 379 & $117-3281$ & 512 & $138-1366$ & 0.958 \\
\hline \multicolumn{10}{|c|}{ S-BAP (U/l) } \\
\hline Pre & 29.6 & $18.0-70.3$ & 30.0 & $14.3-71.2$ & 25.8 & $16.2-88.6$ & 27.5 & $16.1-63.5$ & \\
\hline Post & 28.8 & $16.7-55.7$ & 30.5 & $13.7-72.8$ & 27.5 & $13.4-73.9$ & 28.2 & $15.3-75.4$ & 0.977 \\
\hline \multicolumn{10}{|c|}{$\mathrm{S}-\mathrm{OC}(\mathrm{ng} / \mathrm{ml})$} \\
\hline Pre & 10.4 & $4.7-16.2$ & 9.8 & $6.4-17.9$ & 9.6 & $4.0-27.7$ & 10.3 & $3.2-15.4$ & \\
\hline Post & 11.2 & $4.4-16.4$ & 9.7 & $6.3-16.3$ & 10.5 & $3.9-24.7$ & 10.1 & $5.9-13.8$ & 0.841 \\
\hline
\end{tabular}

BCE, bone collagen equivalents.

${ }^{\mathrm{a}, \mathrm{b}}$ Values in rows with unlike superscript letters were significantly different (Tukey post-hoc test; $P<0.05$ ).

$*$ Effect of treatment on post-intervention variables was assessed by ANCOVA including age, gender, season and baseline concentrations as covariates.

No significant baseline differences in biochemical variables across the treatment groups were observed. Vitamin D supplementation significantly increased S-25(OH)D concentrations. Vitamin D and/or probiotic supplementation had no effect on serum PTH or bone turnover markers concentrations. In conclusion, probiotics and/or vitamin D supplementation over 4 weeks appear to have no effect on the rate of bone turnover in apparently-healthy adults.

We wish to acknowledge the Irish Department of Agriculture, Food and Fisheries through the Food Institutional Research Measure for their support.

1. Gilman J \& Cashman KD (2006) Curr Issues Intest Microbiol 7, 1-5.

2. Giuliano AR \& Wood, RJ (1991) Am J Physiol 260, G207-G212. 